\title{
TESTE DE VIGOR EM SEMENTES DE SORGO PARA PREDIZER O ARMAZENAMENTO ${ }^{1}$
}

\author{
RAMIRO VILELA DE ANDRADE, JOÃO TITO DE AZEVEDO², \\ CLEVERSON DA SILVEIRA BORBA ${ }^{3}$ e ANTÔNIO CARLOS DE OLIVEIRA ${ }^{3}$
}

\begin{abstract}
RESUMO - $\mathrm{O}$ teste padrão de germinação é realizado em condições adequadas de umidade, temperatura e luminosidade, e por isso não é "per si" um indicativo para o armazenamento seguro e de um bom desempenho das sementes no campo. As transformações degenerativas sofridas pelas sementes, na maioria das vezes não são avaliadas pelo teste de germinação, como são pelos testes de vigor. Por isso, um experimento foi instalado no laboratório de análise de sementes do Centro Nacional de Pesquisa de Milho e Sorgo (CNPMS), com o objetivo de avaliar o vigor em sementes de sorgo e comparar os resultados com a germinação obtida trimestralmente durante dezoito meses de armazenamento, em condições ambientais de armazém convencional.. Sementes de dezoito cultivares foram submetidas ao teste de solução de cloreto de amônio $\left(\mathrm{NH}_{4} \mathrm{Cl}\right)$ e ao teste de envelhecimento precoce. Os resultados foram comparados utilizando-se correlações e distâncias euclidianas entre os testes de vigor e as porcentagens de germinação obtidas em diferentes períodos de armazenamento. Verificaram-se correlações positivas significativas entre as porcentagens de vigor e germinação das sementes na maioria dos períodos de armazenamento. Observou-se também uma maior similaridade, ou seja, uma menor distância euclidiana, entre a germinação obtida aos seis meses de armazenamento e o teste de solução de cloreto de amônio. Quanto ao teste de envelhecimento precoce, essa similaridade ocorreu aos quinze meses de armazenamento. Conclui-se que o teste de solução de cloreto de amônio é mais indicado para predizer o potencial de armazenamento de sementes de sorgo por um período de seis meses, enquanto que o teste de envelhecimento precoce é mais indicado para predizer o armazenamento de semente para um período de quinze meses.
\end{abstract}

Termos de indexação: Sorghum bicolor (L.) Moench, germinação, envelhecimento precoce, armazenamento.

\section{STORABILITY OF SORGHUM PREDICTED BY VIGOR TESTS}

\begin{abstract}
High initial germination values on the standard germination test neither provide a safe indication of a well stored seeds nor assure a good performance under field conditions. Degerative metabolism transformation that may occur in seeds are not evaluated by standard germination tests. Vigoir tests provide better information about the storability of the seed lot. An experiment was carried out on the seed analysis laboratory of the Centro Nacional de Pesquisa de Milho e Sorgo (Nacional Maize and Sorghum Research Center), EMBRAPA, Brazil, to evaluate vigor in sorghum seeds and to compare the results with germination values attained during eighteen months in open storage. Sorghum seeds of eighteen cultivars were tested both by imersion in $\mathrm{NH}_{4} \mathrm{Cl}$ and by accelerated aging test. The results were evaluated by comparing correlations and euclidian distances between values of vigor and standard germination tests under different storage periods and indicated positive and significative correlations between standard germiantion and vigor seed tests with most storage periods. There was the greatest similarity or smallest euclidian distance between standard germination and imersion in $\mathrm{NH}_{4} \mathrm{Cl}$ at six months storage, while the greatest similarty between acelerated aging test and storage period was attained at fifteen months of storage.
\end{abstract}

Index terms: Sorghum bicolor (1.) Moench., germination, accelerated aging, storage.

${ }_{1}^{1}$ Aceito para publicação em 05.08.93.

${ }^{2}$ Pesquisador, M.Sc. EMBRAPA/Centro Nacional de Pesquisa de Milho e Sorgo, Sete Lagoas, MG, CEP 35701-970, Caixa Postal 151.

${ }^{3}$ Pesquisador, PhD. EMBRAPA/Centro Nacional de Pesquisa de Milho e Sorgo.

\section{INTRODUÇÃO}

O conhecimento prévio do potencial de armazenamento de um lote de sementes é muito importante para a indústria sementeira. Os lotes de sementes de me- nor vigor, desde que dentro dos padrões, devem ser comercializados primeiramente, deixando aqueles de maior vigor para serem comercializados numa etapa seguinte, uma vez que esses, geralmente, apresentam um maior potencial de arma- 
zenamento.

O teste padrão de germinação "per si" não oferece um indicativo confiável para um armazenamento seguro, uma vez que a sua realização se dá sob condições ótimas de umidade, temperatura e luminosidade. As transformações degenerativas mais sutis não avaliadas pelo teste de germinação, exercem grande influência no potencial de desempenho das sementes com reflexos na capacidade de armazenamento, na emergência no campo, no crescimento e produtividade das plantas (Popinigis, 1977). Essas transformações podem ser detectadas e avaliadas através do teste de vigor, que irá fornecer um indicativo do potencial de armazenamento das sementes.

Existe uma série de testes para se avaliar o vigor em sementes, mas nenhum deles vem sendo adotado universalmente. Para o sorgo, geralmente são utilizados os testes de envelhecimento precoce (Delouche \& Baskin, 1973), o de frio modificado (Hoppe, 1955), o de solução tóxica (Vanderlip et al., 1973) e índice de velocidade de emergência (Maguire, 1962).

Existem vários trabalhos de vigor em sementes de sorgo correlacionando os resultados com a emergência no campo (Petrini et al., 1988; Yayock et al., 1975; Abdulahi \& Vanderlip, 1972; Camargo, 1971; Ahmed, 1977). No Brasil, a literatura sobre o vigor de sementes de sorgo e suas relações com o armazenamento é escassa. Souza \& Marcos Filho (1975), testaram vários métodos para se determinar o vigor das sementes de sorgo e verificaram que a eficiência dos diferentes métodos estudados depende da qualidade das sementes. Concluíram também que os testes de imersão em solução de cloreto de amônio e a velocidade de germinação foram mais eficientes para a avaliação do vigor. Miranda (1967) armazenou, durante um ano, sementes de três cultivares de sorgo granífero em condições de ambiente não controlado e em câmara seca (45 a $60 \%$ de umidade relativa do ar) e verificou que as sementes armazenadas em ambiente controlado mantiveram a germinação, enquanto que aquelas conservadas em condições de ambiente não controlado acusaram uma queda acentuada na germinação sendo o período de fevereiro a agosto o mais prejudicial às sementes nas condições de Recife. Este trabalho teve por objetivo avaliar o vigor de sementes de sorgo para predizer seu potencial de armazenamento durante seis diferentes períodos.

\section{MATERIAL E MÉTODOS}

Foram utilizadas as sementes das cultivares de sorgo, BR 005-R, BR 008-B, BR 301, BR 304, BR 506, BR 507, BR 602, CMS x S-743, CMS x S749, CMS x S-739, CMS x S-353, CMS x S-354, CMS x S-356, CMS x S-362, CMS x S-365, CMS x S-366, CMS x S-368 e CMS x S-369. Foi utilizada uma amostra de aproximadamente $4,0 \mathrm{~kg}$ de cada cultivar que, após ter sido homogeneizada em um homogeneizador tipo Gamet, foi dividida em quatro sub-amostras.

Para realização do teste envelhecimento precoce, utilizaram-se a câmara tradicional, e a câmara de crescimento de microorganismos tipo B.O.D.. Quatrocentas sementes de cada subamostra foram colocadas em caixas plásticas gerbox, dispostas em uma única camada sobre uma tela metálica, a 2,0 cm do fundo da caixa, contendo $40 \mathrm{ml}$ de água destilada. Cada gerbox constituiu em uma mini-câmara, que foi colocada nas câmaras de envelhecimento precoce (tradicional e B.O.D.) durante 120 horas à temperatura constante de $42^{\circ} \mathrm{C}$ e numa saturação de $100 \%$ de umidade relativa do ar.

Quatrocentas sementes de sorgo de cada sub amostra foram também submetidas em imersão em solução de cloreto testes de imersão em solução de cloreto de amônio e a velocidade de germinação foram mais eficientes para a avaliação do vigor. Miranda (1967) armazenou, durante um ano, sementes de três cultivares de sorgo granífero em condições de ambiente não controlado e em câmara seca (45 a $60 \%$ de umidade relativa do ar) e verificou que as sementes armazenadas em ambiente controlado mantiveram a germinação, enquanto que aquelas conservadas em condições de ambiente não controlado acusaram uma queda acentuada na germinação sendo o período de fevereiro a agosto o mais prejudicial às sementes nas condições de Recife. Este trabalho teve por objetivo avaliar o vigor de sementes de sorgo para predizer seu potencial de armazenamento durante seis diferentes períodos.

\section{MATERIAL E MÉTODOS}

Foram utilizadas as sementes das cultivares de sorgo, BR 005-R, BR 008-B, BR 301, BR 304, BR 506, BR 507, BR 602, CMS x S-743, CMS x S-749, CMS x S-739, CMS x S-353, CMS x S-354, CMS x S-356, CMS x S-362, CMS x S365 , CMS x S-366, CMS x S-368 e CMS $x$ S-369. Foi utilizada uma amostra de aproximadamente $4,0 \mathrm{~kg}$ de cada cultivar que, após ter sido homogeneizada em um homogeneizador tipo Gamet, foi dividida em quatro sub amostras.

Para realização do teste envelhecimento precoce, utilizaram-se a câmara tradicional, e a câmara de crescimento de microorganismos tipo B.O.D.. Quatrocentas sementes de cada sub-amostra foram colocadas em caixas plásticas gerbox, dispostas em uma única camada sobre uma tela metálica, a 2,0 cm do fundo da caixa, contendo $40 \mathrm{ml}$ de água destilada. Cada gerbox constituiu em uma mini-câmara, que foi colocada nas câmaras de envelhecimento precoce (tradicional $\mathrm{e}$ B.O.D.) durante 120 horas à temperatura constante de $42^{\circ} \mathrm{C}$ e numa saturação de $100 \%$ de umidade relativa do ar.

Quatrocentas sementes de sorgo de cada sub-amostra foram também submetidas em imersão em solução de cloreto de amônio $\left(\mathrm{NH}_{4}\right) \mathrm{Cl}$ na concentração de $2 \%$ à temperatura de $42^{\circ} \mathrm{C}$, durante 2 horas e a seguir lavadas em água corrente (Yayock et al., 1975).

Após os procedimentos descritos anteriormente, as sementes foram colocadas para germinar, de acordo com as normas prescritas nas Regras de Análises de Se- 
mentes - R.A.S. (Brasil, 1980), porém, fazendo-se uma única leitura no $10^{\circ}$ dia de permanência no germinador. Com as sementes não submetidas aos testes de vigor foram conduzidos testes padrão de germinhação, prescrito nas R.A.S. nos períodos de $0,3,6,9,12,15$ e 18 meses, as quais foram mantidas em armazém convencional, localizado em uma área, onde ocorreram as condições climatológicas de umidade relativa do ar e temperatura, discriminadas na Tabela 4. Com o objetivo de se verificar o grau de concordância entre os porcentuais de germinação obtidos após os testes de vigor (envelhecimento precoce e imersão em cloreto de amônio) e os mesmos porcentuais obtidos após os diferentes períodos de armazenamento, foram determinadas as distâncias euclidianas para as diferentes situações. $\mathrm{O}$ procedimento consistiu na utilização da seguinte expressão:

$d_{i i}=\left[\sum_{j=1}^{18}\left(\chi_{i j}-\chi_{i j}^{\prime}\right)^{2}\right]^{1 / 2}$

onde $\mathrm{d}_{\mathrm{i}} \mathrm{i}^{\prime}$ é a distância euclidiana entre $\mathrm{o}$ i-ésimo teste de vigor e o i-ésimo período de armazenamento; $x_{i}$ jé o porcentual de germinação de j-ésima $(j=1,2, \ldots$. 18) obtido no i-ésimo teste de vigor e $x_{i}$ jé o porcentual de germinação de j-ésima cultivar obtido no i-ésimo período de armazenamento. Conforme se pode deduzir da expressão anterior, menores distâncias indicam resultados mais concordantes.

A instalação do experimento foi realizada de acordo com delineamento experimental inteiramente casualizado com 18 cultivares e quatro repetições.

\section{RESULTADOS E DISCUSSÃO}

Os resultados dos testes de germinação e vigor das sementes das dezoito cultivares de sorgo estão na Tabela 1. As cultivares de sorgo apresentaram qualidades fisiológicas das sementes bastante variadas, uma vez que foram produzidas em épocas e locais diferentes, embora fossem do mesmo ano. As sementes das cultivares mais vigorosas, apresentaram um bom potencial de armazenamento, enquanto que naquelas de menor vigor houve uma acentuada redução no potencial de armazenamento, notadamente naquele previsto pelo teste de envelhecimento precoce. Esses resultados são concordantes com Petrini et al. (1988), os quais salientaram a sensibilidade desse teste com relação a qualidade fisiológica das sementes de sorgo, ou seja, sementes de alta qualidade fisiológica mostraramse poucos sensíveis ao efeito da temperatura e período de exposição só diferindo estatisticamente da emergência no campo, quando expostas à temperatura de $45^{\circ} \mathrm{C}$ durante 120 horas. Por outro lado, concluiram os autores, que em sementes que apresentaram baixa qualidade, o envelhecimento precoce reduziu a germinação a níveis significativos comparados com a emergência no campo. Delouche \& Baskin (1973), constataram que lotes de sementes que mantiveram alta germinação após o teste de envelhecimento precoce, apresentavam um bom potencial de armazenamento, enquanto que aqueles que reduziram substancialmente a germinação durante o teste, não se conservaram bem e a germinação decresceu rapidamente durante o armazenamento. No presente trabalho, o teste de envelheci-

\begin{tabular}{|c|c|c|c|c|c|c|c|c|c|c|}
\hline \multirow{3}{*}{ Cultivar } & \multicolumn{3}{|c|}{ Teste de Vigor } & \multicolumn{7}{|c|}{ Teste de Germinação } \\
\hline & \multirow{2}{*}{$\begin{array}{c}\text { EP Câmara } \\
\text { Tradicional } \\
\%\end{array}$} & \multirow{2}{*}{$\begin{array}{c}\text { EP Câmara } \\
\text { B.O.D. } \\
\%\end{array}$} & \multirow{2}{*}{$\begin{array}{c}\text { Solução } \\
\mathrm{NH}_{4} \mathrm{Cl} \\
\%\end{array}$} & \multicolumn{7}{|c|}{ Armazenamento(meses) } \\
\hline & & & & $\begin{array}{l}00 \\
\%\end{array}$ & $\begin{array}{l}03 \\
\%\end{array}$ & $\begin{array}{l}06 \\
\%\end{array}$ & $\begin{array}{l}09 \\
0\end{array}$ & $\begin{array}{l}12 \\
\%\end{array}$ & $\begin{array}{l}15 \\
\%\end{array}$ & 18 \\
\hline BR $005 \mathrm{R}$ & 62 & 49 & 77 & 80 & 79 & 75 & 70 & 67 & 58 & 50 \\
\hline BR.008 B & 67 & 62 & 83 & 86 & 86 & 79 & 80 & 75 & 66 & 65 \\
\hline BR 301 & 51 & 40 & 68 & 74 & 71 & 69 & 68 & 62 & 62 & 56 \\
\hline BR 304 & 67 & 75 & 76 & 83 & 77 & 76 & 77 & 67 & 66 & 60 \\
\hline BR 506 & 59 & 59 & 69 & 73 & 69 & 65 & 64 & 60 & 51 & 51 \\
\hline BR 507 & 66 & 70 & 81 & 88 & 82 & 78 & 77 & 69 & 57 & 60 \\
\hline BR 602 & 60 & 50 & 75 & 75 & 74 & 72 & 75 & 67 & 63 & 67 \\
\hline CMS x S 743 & 40 & 30 & 60 & 73 & 65 & 55 & 61 & 50 & 45 & 40 \\
\hline CMS x S 749 & 48 & 46 & 70 & 79 & 75 & 65 & 70 & 60 & 52 & 39 \\
\hline CMS x S 739 & 36 & 28 & 52 & 62 & 60 & 58 & 56 & 46 & 42 & 50 \\
\hline CMS x S 353 & 82 & 81 & 88 & 96 & 91 & 90 & 91 & 87 & 82 & 80 \\
\hline CMS x S 354 & 59 & 65 & 72 & 81 & 74 & 73 & 77 & 68 & 60 & 60 \\
\hline CMS x S 356 & 44 & 38 & 64 & 73 & 68 & 65 & 66 & 61 & 52 & 54 \\
\hline CMS x S 362 & 56 & 58 & 73 & 80 & 79 & 75 & 79 & 66 & 62 & 60 \\
\hline CMS x S 365 & 44 & 48 & 71 & 89 & 83 & 72 & 77 & 68 & 63 & 62 \\
\hline CMS x S 366 & 43 & 44 & 56 & 82 & 72 & 68 & 77 & 61 & 44 & 53 \\
\hline CMS x S 368 & 52 & 50 & 61 & 71 & 70 & 66 & 71 & 61 & 55 & 38 \\
\hline CMS x S 369 & 50 & 42 & 59 & 59 & 57 & 56 & 54 & 45 & 41 & 48 \\
\hline
\end{tabular}

mento precoce provocou um declínio acentuado na germinação das sementes, mas não a ponto de reduzí-la a níveis tão baixos como os encontrados por Souza \& Marcos Filho (1975), os quais salientaram a drasticidade do teste em sementes de sorgo, notadamente no período de sete dias de exposição à temperatura de $42^{\circ} \mathrm{C}$, impedindo praticamente a sua germinação. Os porcentuais de germinação obtidos após o teste de envelhecimento precoce (Tabela 2) apresentaram correlação positiva e significativa ao nível de $5 \%$ de probabilidade, com os mesmos porcentuais obtidos a partir do $6^{\circ}$ mês de armazenamento das sementes até o $15^{\circ}$, com exceção da câmara E.P. aos nove meses. No caso do teste de imersão em cloreto de amônio esses porcentuais de germinação também apresentaram correlação positiva e significativa para todos os períodos de armazenamento, com exceção do $18^{\circ}$ mês. Esses resultados são concordantes com aqueles encontrados por Delouche \& Baskin (1973), os quais encontraram altas correlações positivas entre os porcentuais de germinação obtidos após o teste de envelhecimento precoce aplicados a vários lotes de sementes de sorgo, e

\section{TABELA 1. Vigor obtido por diferentes métodos e germinação de sementes de \\ diversas cultivares de sorgo armazenadas por vários meses. Sete Lagoas - MG, 1992.}


TABELA 2. Coeficientes de correlação simples obtidos através de diferentes testes de vigor com a germinação obtida em diferentes períodos de armazenamento. Sete Lagoas, MG, 1992.

\begin{tabular}{lcccccc}
\hline & \multicolumn{5}{c}{ Armazenamento (meses) } \\
\cline { 2 - 7 } & \multicolumn{1}{c}{03} & 06 & 09 & 12 & 15 & 18 \\
\hline Câmara EP $42^{\circ} \mathrm{C}, 100 \%$ UR, $120 \mathrm{~h}$ & 0.720 & $0.847^{* *}$ & 0.685 & $0.806^{* *}$ & $0.785^{* *}$ & 0.415 \\
B.O.D. EP 42 ${ }^{\circ} \mathrm{C}, 100 \%$ UR, $120 \mathrm{~h}$ & 0.727 & $0.849^{* *}$ & $0.764^{* *}$ & $0.799^{* *}$ & $0.738^{* *}$ & 0.333 \\
Solução Tóxica NH $\mathrm{Cl}, 40^{\circ} \mathrm{C}, 2 \mathrm{~h}$ & $0.873^{* *}$ & $0.892^{* *}$ & $0.756^{*}$ & $0.879^{* *}$ & $0.863^{* *}$ & 0.390 \\
\hline
\end{tabular}

**; * correlação significativa a $1 \%$ e $5 \%$, respectivamente.

TABELA 3. Distâncias euclidianas em relação a porcentagem de germinação, obtidas entre diferentes períodos de armazenamento e métodos de envelhecimento aplicados em 18 cultivares de sorgo. Sete Lagoas MG, 1992.

\begin{tabular}{lrrccccc}
\hline \multirow{2}{*}{$\begin{array}{c}\text { Métodos } \\
\text { de }\end{array}$} & \multicolumn{6}{c}{ Períodos de armazenamento (meses) } \\
\cline { 2 - 7 } \multicolumn{1}{c}{ Envelhecimento } & 00 & \multicolumn{1}{c}{03} & 06 & 09 & 12 & 15 & 18 \\
\hline E.P. (Câmara tradicional) & 105.73 & 88.29 & 69.48 & 79.64 & 45.90 & 31.03 & 67.40 \\
E.P. (B.O.D.) & 119.25 & 103.41 & 85.10 & 93.19 & 61.45 & 46.42 & 76.03 \\
Solução de $\mathrm{NH}_{4} \mathrm{Cl}$ & 43.26 & 26.44 & 18.12 & 28.31 & 34.27 & 59.43 & 99.94 \\
\hline
\end{tabular}

TABELA 4. Umidade relativa do ar (\%) e temperatura $\left({ }^{\circ} \mathrm{C}\right)$ médias mensais do período de Janeiro de 1990 a Maio de 1991. Sete Lagoas - MG, 1992.

\begin{tabular}{lcc}
\hline $\begin{array}{c}\text { Mês/ } \\
\text { ano }\end{array}$ & $\begin{array}{c}\text { Umidade relativa } \\
(\%)\end{array}$ & $\begin{array}{c}\text { Temperatura } \\
\left({ }^{\circ} \mathrm{C}\right)\end{array}$ \\
\hline Jan/90 & 65 & 23.6 \\
$\mathrm{Fev} / 90$ & 76 & 23.2 \\
$\mathrm{Mar} / 90$ & 74 & 23.6 \\
$\mathrm{Abr} / 90$ & 78 & 22.8 \\
$\mathrm{Mai} / 90$ & 70 & 19.4 \\
$\mathrm{Jun} / 90$ & 69 & 18.8 \\
$\mathrm{Jul} / 90$ & 68 & 18.1 \\
Ago/90 & 66 & 18.7 \\
$\mathrm{Set} / 90$ & 61 & 20.4 \\
Out/90 & 61 & 23.0 \\
Nov/90 & 67 & 23.9 \\
Dez/90 & 72 & 22.9 \\
Jan/91 & 84 & 22.1 \\
Fev/91 & 78 & 23.2 \\
Mar/91 & 78 & 22.6 \\
Abr/91 & 74 & 21.3 \\
Mai/91 & 75 & 19.1 \\
\hline
\end{tabular}

os porcentuais de germinação obtidos após o armazenamento em condições ambientais do estado do Mississippi, E.U.A.. Quanto ao teste de imersão em solução de $\left(\mathrm{NH}_{4}\right) \mathrm{Cl}$, Vanderlip et al (1973), Yayock et al. (1973) encontraram altos coeficientes de correlação entre os porcentuais de germinação e o estabelecimento de plântulas no campo, principalmente quando a concentração de cloreto de amônio foi de $1 \%$ a $4 \%$ e a temperatura variando de $20^{\circ} \mathrm{C}$ a $30^{\circ} \mathrm{C}$. Delouche \& Baskin (1973) também mencionaram a drasticidade do teste de envelhecimento precoce em sementes e sugeriram que se fizesse o ajuste do teste (temperatura e tempo de exposição) a fim de predizer o período desejado de armazenamento de um lote de sementes.

A Tabela 3 apresenta as distâncias euclidianas, em relação aos porcentuais de germinação, obtidos entre os testes de vigor e os períodos de armazenamento das sementes. Observa-se que para o teste distâncias ocorreram aos quinze meses de armazenamento, em ambas as câmaras (tradicional e B.O.D.). Esse resultado de envelhecimento precoce as menores indica que, dentre os períodos de armazenamento estudados, o período de 15 meses foi o que apresentou os porcentuais de germinação mais próximos daqueles obtidos nos testes de envelhecimento precoce, ou seja, foi o período que apresentou resultados mais concordantes com os testes de vigor. No caso do teste de imersão em solução de cloreto de amônio, a menor distância ocorreu no período de seis meses de armazenamento.

\section{CONCLUSÕES}

Nas condições em que o trabalho foi realizado, concluiu-se que o teste de imersão em solução de cloreto de amônio prediz a porcentagem de germinação das sementes após um período de armazenamento de cerca de seis meses, enquanto que o teste de envelhecimento precoce pode predizer o poder germinativo de sementes dessa espécie após um período de armazenamento em torno dos quinze meses.

\section{REFERÊNCIAS}

ABDULAHI, A.; VANDERLIP, R.L. Relationship of vigor tests and seed source and size to sorghum seedling establishment. Agronomy Journal, 64 (2): 143-4, 1972.

AHMED, H.D. Evaluating differences in vigor amongst sorghum seed lots by various test methods. Seed Research, 5: 152-7, 1977.

BRASIL. Ministério da Agricultura. Equipe Técnica da divisão de sementes e mudas. Regras para análises de sementes. Brasília, 1980. 188p.

CAMARGO, C.P. Effect of seed vigor upon field performance and yield of grain sorghum (Sorghum bicolor (L.) Moench. Missipi State University, 1971. 61p. (M.S.U. Dissertação Mestrado).

DELOUCHE, J.C.; BASKIN, C.C. Accelerated aging techniques for predicting the relative storability of seed lots. Seed Science and Technology, 1(2):427-455, 1973.

HOPPE, P.E. Cold testing for seed corn. Wiscosin Agriculture Experiment Station, 
1955. 5p. (Bulletin 507).

MAGUIRE, S.D. Speed of germination-aid in selection and evaluation for seedling emergence and vigor. Crop Science, 2 (2):176-7, 1962.

MIRANDA, A. Conservação de sementes de sorgo. Boletim Técnico Instituto Pesquisa Agropecuária IPA, 21: 27-33, 1967.

PETRINI, J.A; FERNANDEZ, D; ZONTA, E.P. Testes de vigor para predizer a emergência de sorgo. Pesquisa Agropecuária Brasileira, 23(6):593-8, 1988.

POPINIGIS, F. Fisiologia da semente. Brasília: AGIPLAN, 1977, 289 p.

SOUZA, F.H. de; MARCOS FILHO, J. Estudo comparativo de métodos para a avaliação do vigor de sementes de sorgo (Sorghum vulgare Pers), Anais da Escola Superior Luiz de Queiróz, 33:36983, 1975.

VANDERLIP, R.L.; MOCKEL, F.E.; HALIN, J. Evaluation of vigor tests for sorghum seed. Agronomy Journal, 65:4868, 1973.

YAYOCK, J.; HALIN, J.; VANDERLIP, R.L. Temperature time and $\left(\mathrm{NH}_{4}\right) \mathrm{Cl}$ concentration in vigor testing of sorghum seed. Agronomy Journal, 67(2):241-2, 1975. 